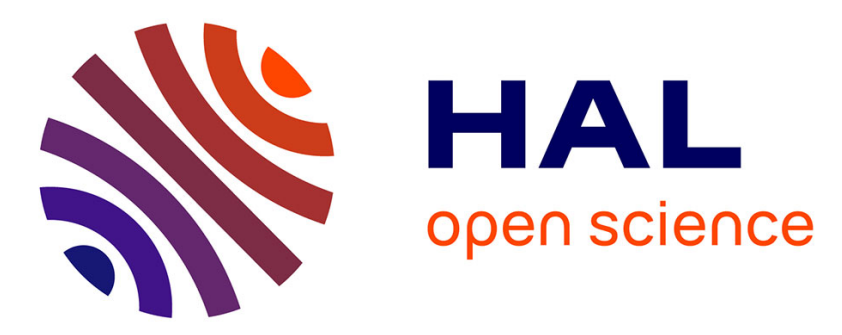

\title{
Characterising the genetic basis of immune response variation to identify causal mechanisms underlying disease susceptibility
}

\author{
Maxime Rotival
}

\section{- To cite this version:}

Maxime Rotival. Characterising the genetic basis of immune response variation to identify causal mechanisms underlying disease susceptibility. HLA: Immune Response Genetics, 2019, 94 (3), pp.275284. 10.1111/tan.13598 . pasteur-02868915

\section{HAL Id: pasteur-02868915}

\section{https://hal-pasteur.archives-ouvertes.fr/pasteur-02868915}

Submitted on 17 Jun 2020

HAL is a multi-disciplinary open access archive for the deposit and dissemination of scientific research documents, whether they are published or not. The documents may come from teaching and research institutions in France or abroad, or from public or private research centers.
L'archive ouverte pluridisciplinaire $\mathbf{H A L}$, est destinée au dépôt et à la diffusion de documents scientifiques de niveau recherche, publiés ou non, émanant des établissements d'enseignement et de recherche français ou étrangers, des laboratoires publics ou privés. 


\section{Characterising the genetic basis of immune response variation to} identify causal mechanisms underlying disease susceptibility

Short title: genetic basis of immune response variation

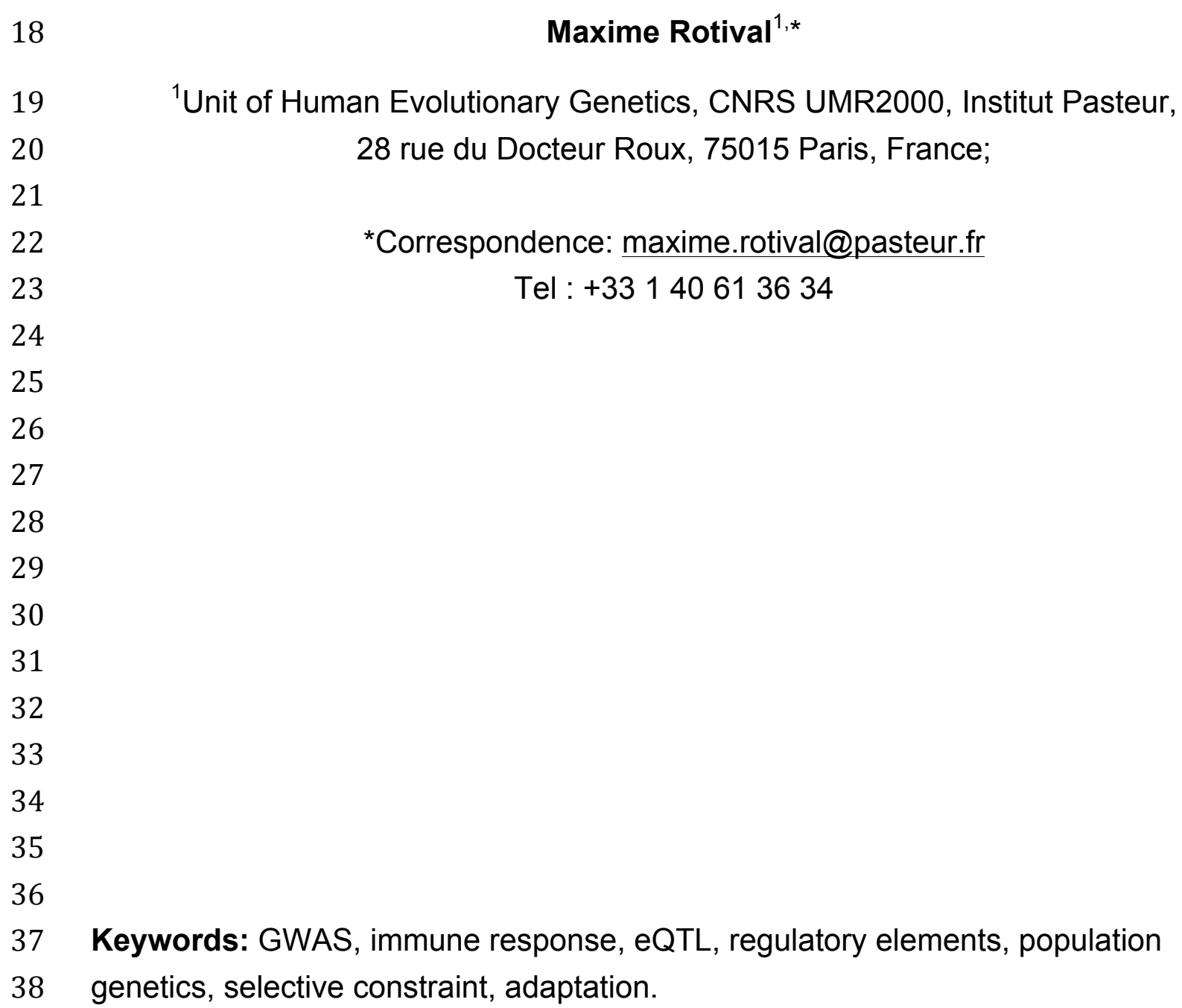

37 Keywords: GWAS, immune response, eQTL, regulatory elements, population 


\section{Abstract (250 words)}

40 Over the last ten years, genome-wide association studies (GWAS) have identified

41 hundreds of susceptibility loci for autoimmune diseases. However, despite increasing

42 power for the detection of both common and rare coding variants affecting disease

43 susceptibility, a large fraction of disease heritability has remained unexplained. In

44 addition, a majority of the identified loci are located in non-coding regions, and

45 translation of disease-associated loci into new biological insights on the aetiology of

46 immune disorders has been lagging. This highlights the need for a better

47 understanding of non-coding variation and new strategies to identify causal genes at

48 disease loci. In this review, I will first detail the molecular basis of gene expression

49 and review the various mechanisms that contribute to alter gene activity at the

50 transcriptional and post-transcriptional level. I will then review the findings from 10

51 years of functional genomics studies regarding the genetics on gene expression, in

52 particular in the context of infection. Finally, I will discuss the extent to which genetic

53 variants that modulate gene expression at transcriptional and post-transcriptional

54 level contribute to disease susceptibility and present strategies to leverage these

55 information for the identification of causal mechanisms at disease loci in the era of

56 whole genome sequencing. 
60 The host immune response to stress exhibits considerable variation, both at the

61 individual and population levels ${ }^{1}$. While many non heritable factors contribute to this

62 variability, including age, gender or past exposures to pathogens ${ }^{2-5}$ (see ${ }^{1}$ for a review

63 on these aspects). There is also widespread evidence that genetics plays a

64 significant role in determining the efficacy of the human immune response, in

65 particular in the context of innate immunity ${ }^{6-12}$. Genome-wide association studies

66 (GWAS) have been a considerable asset in defining the genomic regions involved in

67 immune disease susceptibility ${ }^{13}$. However, identifying the causal mechanisms

68 through which GWAS variants contribute to disease susceptibility has proven to be

69 extremely challenging. Indeed, it was soon realized that most GWAS pointed toward

70 gene deserts or non-coding regions ${ }^{14,15}$, highlighting the strong need for

71 characterization of the molecular mechanisms through which such non-coding

72 regions may alter immune disease susceptibility ${ }^{16}$. Furthermore, while large GWAS

73 meta-analyses have granted increasing power to capture the effect of common

74 variants on disease susceptibility, a large fraction of disease heritability has

75 remained unaccounted for ${ }^{17}$. There is now increasing evidence to suggest that rare

76 variants, non-coding in particular, may contribute to explain this missing heritability

$77^{18-20}$. Therefore, there is a growing need to understand how non-coding variants, 
78 whether rare or common, contribute to the variability of immune phenotypes in

79 humans. In this review, I will first present the various mechanisms though which non-

80 coding elements may contribute to regulate gene expression, either at the

81 transcriptional level, or post-transcriptionally through RNA splicing and degradation.

82 Then, I will review current knowledge of how common genetic variants drive immune

83 gene expression at the transcriptional and post-transcriptional level. Finally, I will

84 discuss strategies to decipher the impact of rare regulatory variants on immune

85 response and characterize the causal mechanisms that determine immune disease

86 susceptibility.

88 The molecular bases of gene expression

89 In multi-cellular organisms, tight regulation of gene expression programs is essential.

90 First, during development, cells must coordinate expression of various transcription

91 factors to divide and differentiate into future organs. Then, in adult life, regulatory

92 sequence need to allow for cell-type specific gene expression profiles, that are both

93 (i) robust to genetic variations and (ii) able to react appropriately to external stimuli.

94 In human, such combination of resilience and adaptability is achieved through a

95 network of regulatory features, the complexity of which largely exceeds that of the

96 protein coding genome. Indeed, while coding sequence represent only $1.5 \%$ of the 
97 human genome, there is evidence that at least $20 \%$ of the genome is covered by

98 regulatory features, and $10 \%$ is actively bound by known transcription factors ${ }^{21}$.

100 Promoters and enhancers shape transcriptional activity across tissues

101 The regulatory features that control transcription are characterized by epigenetic

102 histone modifications (see Table 1 and ref. ${ }^{22}$ ) and can be decomposed in two main

103 categories (Figure 1a). At the transcription start site, promoters recruit RNA

104 polymerase, along with a variety of other transcription factors, to initiate gene

105 transcription ${ }^{15,23,24}$. While $96 \%$ of promoters are active in more than one tissue or cell

106 type, only $\sim 20 \%$ are ubiquitously expressed indicative of strong tissue-specificity of

107 gene regulation ${ }^{23}$. Tissue-specificity is further increased by distal enhancer regions

108 - located up to $1 \mathrm{Mb}$ away from the promoters they regulate - which, when

109 activated by tissue-specific transcription factors, are brought in contact with their

110 associated promoter by looping of the DNA molecule (Figure $\mathbf{1 b}, \mathbf{c}$ ) to modulate their

111 activity ${ }^{15,24,25}$. Activation of both promoters and enhancers is associated to an

112 opening of the chromatin and acetylation of H3K27 histone residues (Figure 1d,e),

113 together with transcription of non-coding RNAs ${ }^{22,25,26}$. This transcription occurs either

114 in the opposite direction of transcriptional elongation at promoters (upstream

115 antisense RNAs), or bi-directionally for enhancers (enhancer RNAs). Although such 
116 RNAs are rapidly degraded by exosomes, they allow precise quantification of

117 enhancer/promoter activity by sequencing techniques that target the 5 ' end of

118 transcripts such as CAGE-seq or GRO-Cap ${ }^{23,25,27 .}$

119 Over the last 10 years, large Consortia - such as Fantom5 $5^{23,25}, \mathrm{ENCODE}^{26}$,

120 BluePrint ${ }^{28-31}$ or Roadmap Epigenomic ${ }^{22}$ — have combined these techniques with

121 Chromatin immuno-precipitation (ChipSeq) and chromatin accessibility Assays

122 (DNAse-Seq, FAIRE-Seq, or ATAC-Seq) to precisely map promoters and enhancers

123 along the genome and characterize of their activity across tissues. In doing so,

124 theses studies have provided valuable insights on the regulatory architecture of the

125 response to immune stimuli. Notably, immune enhancers were shown to have a

126 distinct sequence composition, DNA structure and activity patterns ${ }^{32}$, with their

127 activation preceding that of their proximal promoters during immune response,

128 revealing their crucial role in the early transcriptional response to immune stimuli ${ }^{33}$.

\section{Three-dimensional chromatin conformation orchestrates transcription}

131 Because enhancers are located far from the genes they regulate,

132 understanding how they are brought into contact with their target gene is of primary

133 importance to decipher the complex networks of that underlies transcriptional

134 regulation in humans. Chromosome Conformation Capture techniques $(\mathrm{Hi}-\mathrm{C}$, and 
135 Promoter-capture Hi-C, Chia-PET) have recently enabled the detection of DNA-DNA

136 interactions along the genome ${ }^{30,31,34,35}$, allowing the assignment of enhancers to their

137 target genes. In practice, a single gene is often regulated by multiple enhancers, and

138 one enhancer can regulate multiple genes ${ }^{15,24}$. Notably, $\sim 70 \%$ of these interactions

139 are restricted to specific regions, known as Topologically associating domains or

140 TADs, which can spread across hundreds of kilobases ${ }^{24,31}$. The boundaries of these

141 TADs are genetically encoded - with evidence of TADs being modified by targeted

142 deletions and genetic variants occurring at the boundary ${ }^{35,36}$ - and are enriched in

143 are binding sites of the insulating factor CTCF. Note, however, that CTCF binding

144 sites have also been observed within TADs ${ }^{35}$, indicating that CTCF binding is not

145 sufficient to induce a TAD boundary. Growing evidence suggests that TADs act to

146 facilitate efficient and insulated co-regulation of functionally related genes. In

147 particular, they were reported to segregate promoters of immune gene together to

148 facilitate their epigenetic priming ${ }^{37}$.

149 Within TADs, promoter/enhancer conformation exhibits a wide variability

150 across tissues, and was proposed to underlie cell type-specificity of enhancer

151 functions ${ }^{31}$. In particular, several studies have reported the existence of enhancers

152 acting as hubs for chromatin-chromatin interactions, linking promoters to other

153 enhancers ${ }^{30,38,39}$. These hubs - sometimes referred as super-enhancers ${ }^{38}$, 
154 frequently interacting regions ${ }^{30}$ or initiator enhancers ${ }^{39}$ - are tissue-specific ${ }^{30}$,

155 preferentially found next to cell identity genes ${ }^{30,38}$ and enriched in tissue-specific

$156 \mathrm{eQTL}^{39}$. More importantly, super enhancers are strongly enriched in disease-related

157 SNPs and largely contribute to auto-immunity ${ }^{30,38}$. For instance, up to $22 \%$ of SLE

158 GWAS loci fall into B-cell specific super enhancers, with disease-linked super-

159 enhancers being observed at key immune genes such as HLA-DRB1, HLA-DQA1,

160 the interferon response factor IRF5 or the NF-KB inhibitor TNFAIP $3^{38}$.

161

\section{Post-transcriptional regulation through alternative splicing}

163 Beyond transcription, the control of gene expression is also ensured at the

164 post-transcriptional level through alternative splicing (AS) and miRNA-mediated

165 degradation. AS can change the function of the protein isoform that is being

166 expressed, through addition/removal of exons encoding functional domains. The

167 most iconic example of such a mechanism is the splicing transition of CD45 antigen

168 during training of adaptive immunity, where T-cells switch from the CD45RO epitope

169 that characterizes naïve T-cells to the CD45RA epitope associated with memory T-

170 cells $^{40}$. AS can also repress gene expression through the introduction of premature

171 stop codons into transcribed sequences, leading to non-sense mediated decay ${ }^{41,42}$.

172 Although our understanding of the splicing code remains incomplete, there is a wide

173 agreement that it involves the recognition by the spliceosome of donor/acceptor 
174 sequences located at the intron boundaries, together with a branchpoint sequence

175 - typically located $\sim 30$ nucleotide upstream of the acceptor splice site ${ }^{41,42}$. This

176 leads to the formation of a lariat and the removal of the intron. AS is induced by

177 competition between splice sites and is regulated through a wide array of RNA

178 binding proteins able to recognize both exonic and intronic regulatory sequences ${ }^{42-}$

17945 . These sequences can act as to either repress or promote, the recruitment of the 180 spliceosome around a specific splice site or exon. In addition, alternative splicing can

181 also be regulated co-transcriptionally, with exon inclusion being associated to a

182 lower transcriptional elongation rate around the alternatively spliced exon ${ }^{41}$.

183

184 Impact of miRNA-mediated degradation on immune response

185 Another layer of post-transcriptional regulation occurs through the binding of

186 miRNAs to 3'UTRs, and the targeting of bound mRNAs to the RNA induced silencing

187 complex (RiSC) ${ }^{46,47}$. The RISC then functions to repress gene expression through

188 degradation of transcripts and inhibition of translation. For example, upon TLR4

189 stimulation, the NF-kB-activated miRNAs mir-146a and miR-155 are strongly up-

190 regulated and bind to 3'UTRs of the TLR4 signal transducers, TRAF6 and IRAK4

191 and the TLR4 inhibitors SHIP1 and SOCS1 ${ }^{48}$, respectively. These mRNAs are then

192 targeted to the RISC for degradation, thereby contributing to the balance of 
193 inflammatory responses to TLR4 stimulation. Several mechanisms can further

194 modulate the impact of miRNAs on gene expression. For instance, usage of

195 alternative UTRs and poly-adenylation sites can change the set of miRNA binding

196 sites available for regulation of gene expression ${ }^{49}$. Such changes have been shown

197 to be widespread upon stimulation ${ }^{49}$ and to play an important role in allowing key

198 regulators of immune response, such as IRF5 or Map Kinases MAPKAP1 and

199 MAP2K4, to escape repression by immune miRNAs ${ }^{49}$. Formation of miRNA isoforms

200 - known as isomiRs - could also contribute to immune response variability.

201 Indeed, it was shown that post-transcriptional modifications of miRNAs through

202 editing of the miRNA seed sequence or shifts in $5^{\prime} / 3^{\prime}$ boundaries of the mature

203 miRNA, can alter their target sites or stability and lead to a decrease in their ability to

204 for regulate mRNAs ${ }^{46,50}$. Furthermore, extensive changes in isomiRs have been

205 observed upon stimulation, including for key immune miRNAs ${ }^{51}$. For instance, upon

206 stimulation by interferon, mir-222 expresses shorter isoforms, which are associated

207 to lower apoptosis ${ }^{52,53}$, highlighting how changes of miRNA isoform can contribute to

208 regulate the response to immune stimuli. 
211 One major challenge of functional genomics is the understanding of the regulatory

212 code that dictates gene expression from the genetic information encoded by DNA

213 sequences. One approach to learn this regulatory code is through the use of

214 naturally-occurring genetic variation. Studies of expression quantitative trait loci

215 (eQTL) aim to identify genetic variants that are associated to gene expression levels

216 in human populations.

\section{Genetic control of transcriptional activity is highly stimulation-specific}

219 eQTL studies have first focused of steady-state expression measurements on

220 lymphoblastoid cell lines or whole blood, from a wide range of populations ${ }^{54-58}$.

221 These studies are revealed that the majority of eQTLs are located in cis, i.e. less

222 than $1 \mathrm{Mb}$ away of the gene they control, and that population-specific eQTLs are

223 driven by changes in allelic frequency rather than gene $x$ gene or gene $x$

224 environment interactions ${ }^{59}$. While a few trans-associations - involving genes

225 located on different chromosomes - were detected, these associations were either

226 weak or driven by cross mapping errors ${ }^{60}$ and changes in cellular composition ${ }^{61}$. The

227 strong potential of eQTL for biomedical applications ${ }^{62}$ has rapidly lead to extend this

228 framework to multiple tissues ${ }^{63}$ or purified immune cell types ${ }^{29,64}$, revealing a strong

229 tissue specificity of eQTLs. Furthermore, exposing human cells to immune and 
230 infectious challenges has allowed the identification of response eQTLs that are

231 associated with changes in gene expression specifically upon immune stimulation

232 (Figure $\mathbf{1 f , g})^{6,9,12,65,66}$. Interestingly, response-eQTLs were found to be highly

233 variable across stimuli and time points ${ }^{8,9,12}$. For instance, the rs9266257 variant was

234 found to control expression of HLA-C in macrophages specifically upon infection by

235 Salmonella, but not Listeria ${ }^{65}$. Interestingly, this phenomenon appeared even more

236 striking among trans-eQTLs which where often revealed by immune stimulation ${ }^{8}$. An

237 outstanding example of this is the TLR1 locus, where the European-specific

238 missense variant rs 5743618 is associated with a decreased inflammatory response

239 to stimulation of the TLR1/2 complex 9 . Interestingly, the European-specific allele of

240 this variant is protective against leprosis $^{67}$ and overlaps one of the strongest

241 signature of natural selection in the human genome ${ }^{9}$, highlighting how selective

242 pressures induced by pathogens can lead to strong inter-individual and inter-

243 population differences in immune phenotypes.

\section{The subtle effect of enhancer variants of gene expression}

246 Studies of the mechanistic bases of eQTLs ${ }^{9,57}$ have shown that promoter variants

247 are largely overrepresented among eQTLs. However, enhancer variant are

248 increasing likely to be found when focusing on secondary, weaker eQTLs ${ }^{63}$. The 
249 increasing power to detect small effect size and highly localized effects offered by 250 eQTL meta-analyses ${ }^{61}$ and analyses of eQTLs at single cell resolution ${ }^{68}$ will likely 251 improve our understanding of the contribution of enhancer variants to gene 252 expression. Another approach to detect subtle effects of enhancers variants on gene 253 expression consists in assaying directly the effect of genetic variants on regulatory 254 activity, based on chromatin accessibility ${ }^{66,69}$, histone modifications ${ }^{29,69}$, or short-live 255 transcriptional products ${ }^{70}$. In doing so, $\mathrm{Li}$ et al. estimated that QTLs for regulatory 256 elements could account for $\sim 60 \%$ of eQTLs, but only $<25 \%$ of enhancers QTLs were 257 associated to an effect on transcription nearest gene ${ }^{69}$, suggesting that only a small 258 fraction of variants that alter regulatory activity lead to a measurable effect on gene 259 expression. However, variants that alter chromatin accessibility with no visible effect 260 on gene expression at basal state can contribute to prime enhancers ${ }^{66}$. Upon 261 stimulation, this priming favours the binding of stimulus-specific transcription factors, 262 such as NF-kB or Stat2, leading to a stronger immune response ${ }^{37,66}$, Together, these 263 observations highlight the potential of eQTL meta-analyses and direct assays of 264 regulatory activity to understand inter-individual variability in immune response. 
266 Genetic regulation of post-transcriptional variability in the immune response

267 Perhaps unsurprisingly given their strong potential to alter protein function,

268 splicing quantitative trait loci (sQTLs, figure 2a) have turned out to be consistently

269 less frequent than eQTLs across the human genome. Indeed, eQTLs appear to be

270 from 1.5 to 6 time more frequent than sQTLs, across studies ${ }^{56,57,58,65,71,72}$ (Table 2).

271 In addition, sQTLs displayed a stronger overlap between conditions of stimulation

272 than eQTLs ${ }^{65,71}$, suggesting a limited impact of stimulation on the genetic regulation

273 of splicing. Nevertheless, disease-causing SQTL have been observed at many key

274 immune genes including OAS1, IRF7, IL7R, IFI44L, TYK2 and ERAP2 ${ }^{65,71,73-75}$,

275 highlighting the importance of considering splicing variation when searching for the

276 causal mechanisms underlying GWAS loci. Interestingly, several of these loci,

277 including OAS1 or ERAP2 present haplotypic signatures of positive and balancing

278 selection, suggesting that these SQTL have conferred a selective advantage to

279 human populations in the past, likely through increased resistance to pathogens. ${ }^{71,73}$.

280 The regulation of miRNA expression has also been extensively studied, both at basal

281 state $^{76,77}$ and in response to infection ${ }^{78}$. Similar to splicing, miRNA QTLs studies

282 (figure $\mathbf{2 b}$ ) have generally suggested a limited genetic control of miRNAs, consistent

283 with expectation of strong selective constraints on miRNA expression due to their 
284 widespread impact on gene expression ${ }^{76,78}$. miRNA QTLs appear to be strongly 285 enriched in variants located in their promoter and eQTLs of their host gene ${ }^{79}$.

286 However, there is also strong evidence that enhancers and loci that are bound by

287 transcription factor (TF) contribute to miRNA expression ${ }^{77}$. Recently, a study has

288 used models of SNP-miRNA interactions to quantify the impact of SNPs on the

289 regulation by miRNAs (reg-eQTL, figure 2c). This has lead to the identification of

$290 \sim 40$ SNP that disrupt miRNA binding sites ${ }^{80}$. The proposed approach, however,

291 suffers from the lack of power usually associated with interaction testing, and may

292 largely underestimate the true number of such reg-eQTL. Larger studies combining

293 expression, genetic and miRNA data are now needed to fully characterize the impact

294 of genetic variants on miRNA-mediated degradation in the immune response.

296 The contribution of regulatory variants to disease susceptibility

297 In support of the importance or eQTLs in disease susceptibility, they have been 298 repeatedly reported to be enriched in disease $\operatorname{loc}^{8,9,12,57,63}$. For instance, the 299 association of the rs 4077515 variant with Inflammatory bowel disease is mediated by 300 activation of a distal enhancer of CARD9 leading to an increase of its transcription,

301 supporting the causal role of CARD9 in Inflammatory bowel disease ${ }^{29}$. Similarly, 302 there is increasing support to the notion that sQTL or mirRNA QTLs can also 
303 contribute to common disease risk $^{69,71,81}$. For instance, the Multiple sclerosis 304 associated variant rs6897932, located within an exon 6 of IL7R, has been shown to 305 promote exon skipping. As a result, a soluble form of IL7R is being produced that is 306 associated with exacerbated auto-immune phenotypes in mice ${ }^{75}$. However, despite 307 evidence that $90 \%$ of auto-immune loci act through regulatory variants, $60 \%$ of which 308 are located at enhancers ${ }^{82}$, it was suggested that only a limited fraction of the QTLs 309 detected at GWAS loci play a causal role in disease susceptibility ${ }^{63,83}$. This 310 observation, which may reflect the robustness of disease gene expression induced

311 by the high redundancy of their enhancers ${ }^{84}$, highlights the importance of taking 312 linkage disequilibrium into account when assessing the co-localization between 313 GWAS loci and eQTLs, or sQTLs ${ }^{85}$.

315 Deciphering the regulatory code to predict the effect of rare variants

316 Recently, the development of massively parallel reporter assays (MPRA) has

317 enabled the direct assessment of cis-regulatory activity associated to specific DNA

318 fragments, either synthetic ${ }^{86}$, or naturally occurring ${ }^{87,88}$. The general principle of

319 MPRA techniques (figure 3) consists in transfecting libraries of barcoded plasmids

320 into live cells and counting the occurrence of each barcode in the transfected cells,

321 as a measure of the activity of the associated regulatory sequence (after 
322 normalization by the barcode frequency in the initial library). By measuring the

323 activity of both alleles at heterozygous loci, these approaches further allow

324 quantifying the impact of genetic variants, or haplotypes, from a single individual.

325 Thus, they constitute a privileged tool for assessing the impact of rare variants on

326 regulatory regions, or identifying causal variants among sets of linked SNPs

327 identified through eQTL analyses ${ }^{88,89}$. For instance, dissection of regulatory

328 mechanisms at a 87 nucleotide-long IFNB1 enhancer indicated that 83 substitutions,

329 out the 261 possible, resulted in altered activity in virus-infected cells ${ }^{88}$.

330 Massively parallel assays have also been used in the context of both splicing ${ }^{90,91}$

331 and miRNA-mediated regulation ${ }^{92,93}$, revealing, for instance, that up to $16 \%$ of splice

332 disrupting variants are located in deep intronic regions ${ }^{90}$. With the development of

333 deep learning frameworks for sequence-based predictions ${ }^{94,95}$, data generated by

334 these assays, will now fuel the construction of predictive models. These models will,

335 in turn, allow quantifying the regulatory impact of novel genetic variants on

336 transcriptional activity ${ }^{88}$, alternative splicing ${ }^{96}$, or miRNA-mediated regulation ${ }^{93}$.

\section{Concluding remarks: a strategy for the future.}

339 Elucidating the mechanisms by which non-coding variants contribute to the inter-

340 individual variability of immune response is now an essential step in order to 
341 translate the findings of epidemiological genetic studies into the clinics ${ }^{13}$. The

342 characterization the human epigenome across a wide variety of tissues and

343 conditions has allowed establishing a first map of the regulatory landscape of the

344 human genome. Further efforts are now needed to fully define the cascade of

345 regulatory events that take place during the immune response, for a wide spectrum

346 of stimuli and with a high temporal resolution. This will allow classifying regulatory

347 elements according to their activity in distinct cell types, and in response to specific

348 stimuli. Then, using heritability partitioning techniques ${ }^{97,98}$ that decompose the

349 genetic variance of complex traits across several SNP categories, we will be able to

350 identify the cell types and stimuli that most strongly contribute to the development of

351 immune disorders. Holistic studies that consider both transcriptional and post-

352 transcriptional aspects of gene expression variation ${ }^{41,66,74}$, and combine RNA seq

353 with sequencing of small RNAs or ATAC-seq, constitute a promising approach to

354 understand the aetiology of immune disorders. Such approaches, however, are only

355 the first step toward the identification of the causal mechanisms that underlie disease

356 susceptibility. Validation of the proposed mechanisms, through colocalization

357 analysis $^{85}$, mendelian randomization approaches ${ }^{99}$ and randomized clinical trials ${ }^{100}$,

358 are then required to formally establish causality. Finally, as whole genome

359 sequencing is becoming increasingly common ${ }^{19}$, the ability to identify rare regulatory 
360 variants at known disease genes will become a crucial step toward personalized

361 treatments of both rare and common immune disorders. In this context, the

362 increased knowledge of the regulatory code granted by functional genomics studies,

363 and MPRA in particular, will constitute a precious tool to predict and assess the

364 effect of rare variants on intermediate disease phenotypes.

365

366 
367 Table 1. Epigenetic marks associated with gene regulation

\begin{tabular}{|c|c|c|}
\hline Epigenetic mark & Associated function & Assay \\
\hline H3K4me1 & Enhancers & Chip-Seq \\
\hline H3K4me3 & Promoters & Chip-Seq \\
\hline $\mathrm{H} 3 \mathrm{~K} 27 \mathrm{Ac}$ & $\begin{array}{c}\text { Active regulatory regions } \\
\text { (promoter/enhancer) }\end{array}$ & Chip-Seq \\
\hline H3K9Ac & $\begin{array}{c}\text { Active regulatory region } \\
\text { (promoter/enhancer) }\end{array}$ & Chip-Seq \\
\hline H3K36me3 & $\begin{array}{l}\text { transcribed regions } \\
\text { /exon definition }\end{array}$ & Chip-Seq \\
\hline H3K9me3 & $\begin{array}{l}\text { repressed region } \\
\text { (heterochromatin) }\end{array}$ & Chip-Seq \\
\hline H3K27me3 & $\begin{array}{l}\text { repressed region } \\
\text { (polycomb) }\end{array}$ & Chip-Seq \\
\hline $\begin{array}{l}\text { Cytosine } \\
\text { methylation }\end{array}$ & $\begin{array}{l}\text { repressed regulatory } \\
\text { regions (promoter/enhancer) }\end{array}$ & BisulfiteSeq \\
\hline Open Chromatin & $\begin{array}{c}\text { Active regulatory region } \\
\text { (promoter/enhancer) }\end{array}$ & $\begin{array}{l}\text { DNAse-Seq/ } \\
\text { ATAC-Seq }\end{array}$ \\
\hline
\end{tabular}

Table 2. Compared number of eQTL and SQTL detected across studies

\begin{tabular}{|c|c|c|c|c|}
\hline Study & Sample used & $\begin{array}{l}\text { Number } \\
\text { of eQTL } \\
\text { detected }\end{array}$ & $\begin{array}{l}\text { Number } \\
\text { of sQTL } \\
\text { detected }\end{array}$ & $\begin{array}{c}\text { Metric } \\
\text { used }\end{array}$ \\
\hline Pickrell, $2010^{56}$ & $\begin{array}{l}\text { lymphoblastoid cell } \\
\text { lines }(n=69)\end{array}$ & 929 & 187 & Exon \\
\hline $\begin{array}{l}\text { Lappalainen, } \\
2013^{57}\end{array}$ & $\begin{array}{l}\text { lymphoblastoid cell } \\
\text { lines }(n=462)\end{array}$ & 3,773 & 639 & Transcript \\
\hline Battle, $2014^{58}$ & Whole blood $(n=922)$ & 10,914 & 2,851 & Transcript \\
\hline Nedelec, $2016^{65}$ & $\begin{array}{c}\text { macrophages } \\
(\mathrm{n}=175 \times 3 \text { conditions })\end{array}$ & 1,647 & 1,120 & Transcript \\
\hline Chen, $2016^{29}$ & $\begin{array}{c}\text { Monocytes, } \\
\text { neutrophils, } \\
\text { CD4+ T-cells }(n=197)\end{array}$ & $\begin{array}{l}6513 \\
5845 \\
5799\end{array}$ & $\begin{array}{l}3044, \\
2034 \\
3347\end{array}$ & $\begin{array}{l}\text { Transcript, } \\
\text { Exon }\end{array}$ \\
\hline Ye, $2018^{71}$ & $\begin{array}{c}\text { Dendritic cells } \\
(3 \text { conditions, } \\
n=99+227+250)\end{array}$ & 6,694 & 2,763 & Transcript \\
\hline
\end{tabular}


a

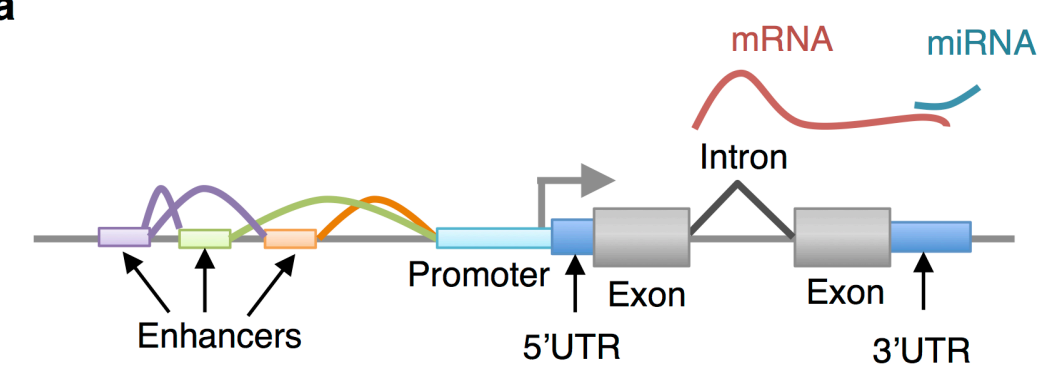

b

Basal state

c Stimulated state
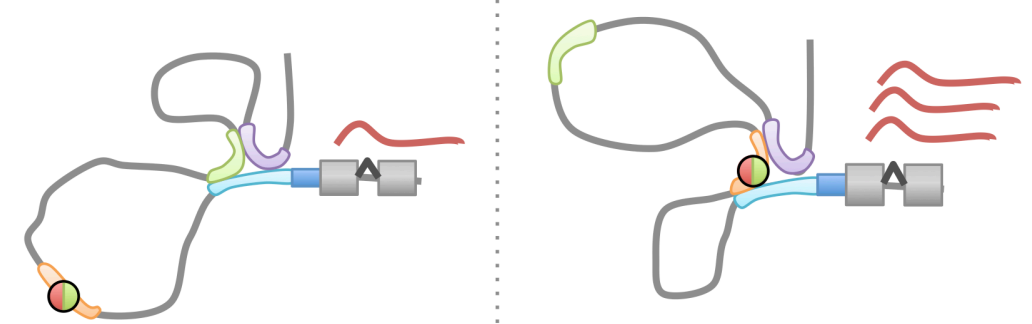

d

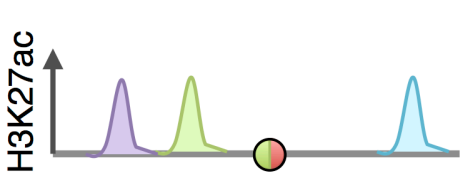

e

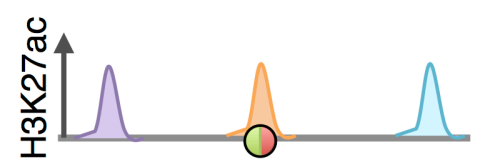

f

g
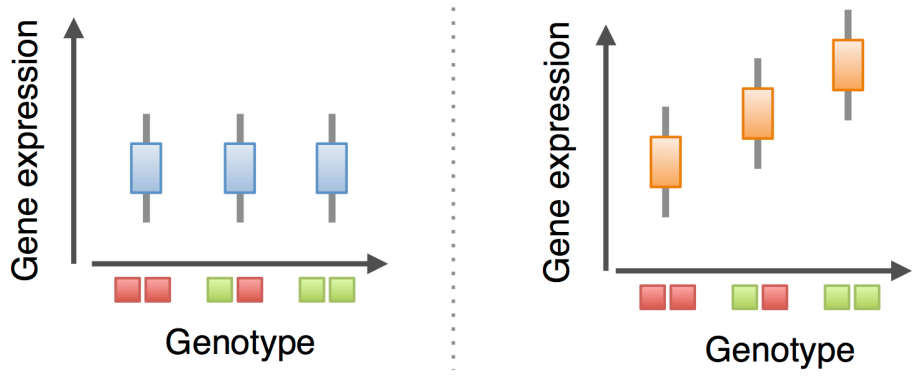

370

371 Figure 1: The molecular bases of response-eQTLs.

a. Genomic dissection of a genic region. Grey arrow represents the transcription start site. Purple, green and orange lines represent possible chromatin-chromatin

374 interactions between enhancers, or between enhancers and the promoter. 
376 code is used throughout the figure. b-c. 3-dimensional representation of

377 chromatin interactions leading to gene expression. At basal state (b), gene

378 expression is mediated by the green enhancer, which comes in contact with the

379 promoter (lightblue) and recruits the purple enhancer. Upon stimulation (c), the

380 green enhancers is sequestrated far from the promoter and replaced by the

381 orange enhancer, allowing the mutation to alter gene expression. d-e. Activity of

382 regulatory elements in the region, at basal state (d) and upon stimulation (e).

383 Peaks indicate H3K27 acetylation with measures activity at promoters and

384 enhancers. f-g. Boxplots of gene expression for each genotype, at basal state (f)

385 and upon stimulation (g). Alleles at the locus are colour coded (red: low

386 expression, green: high expression allele). The impact of the genetic variant

387 located in the orange enhancer is visible only in the stimulated condition, where

388 the enhancer is active. 
a Splice QTL

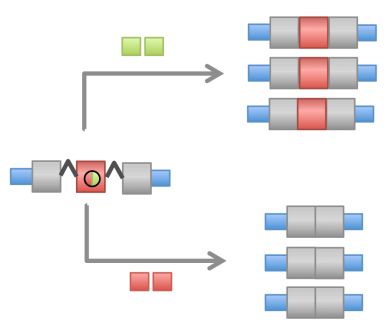

b miRNAQTL

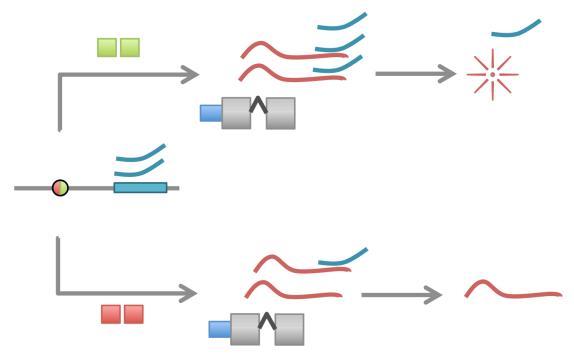

c

Reg-eQTL

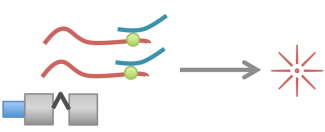

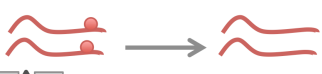

390

Figure 2: Genetic regulation at the post-transcriptional level.

393 a. SQTL alter gene function by changing their splicing, leading to a different protein

394 isoform. b. miRNA QTL that modify the abundance of miRNAs can act in trans in to 395 alter gene expression of genes located elsewhere on the genome through miRNA-

396 mediated degradation. c. SNPs that disturb (or create) miRNA binding sites in the

397 3'UTR of a gene can also change their level of expression, by acting in cis on

398 miRNA-mediated degradation. Such SNPs are referred as reg-eQTLs. 
Create a library of target sequences

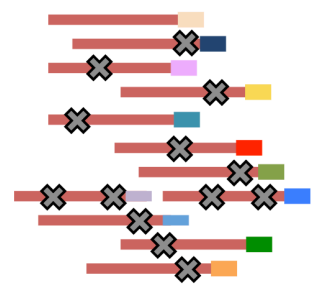

Sequence and count barcodes. Compare to input

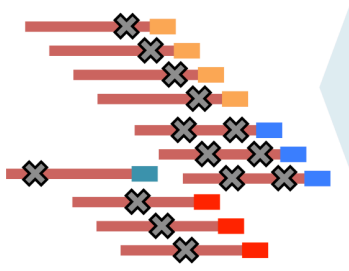

(1)

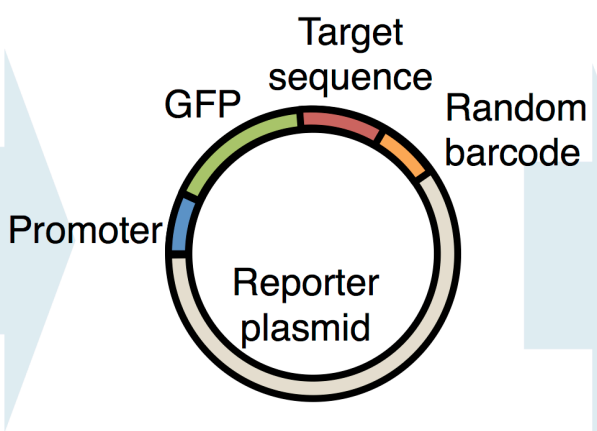

Extract mRNAs

(4)

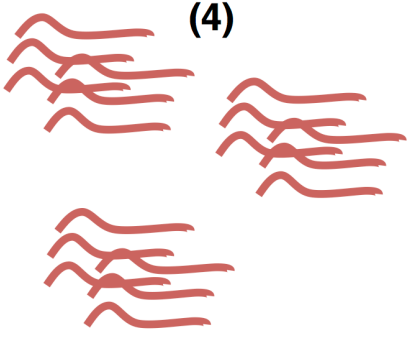

Generate a

Plasmid library

(2)

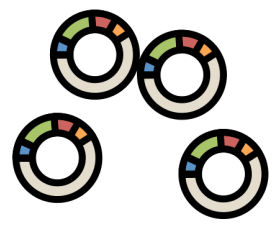

Transfect into target cell type (3)

400

401 Figure 3: Principle of massively parallel reporter assays (MPRA).

402 MPRA consists in forming libraries of putative regulatory sequences, where each

403 sequence is associated to a specific, random barcode (1). The barcoded sequence

404 is then introduced in a reporter plasmid, where it is ligated to the 3' of a green

405 fluorescent protein (GFP) associated with a basic promoter sequence (2). The

406 plasmid library is then transfected into a cell line representative of the tissue of

407 interest (3). RNA from GFP positive cell are harvested and mRNAs of the reporter

408 gene are captured and sequenced (4). The regulatory activity of each sequence is

409 then measured by comparing the counts of its associated barcode in the transfected

410 cell library with that of the initial sequence library (5). 


\section{Acknowledgements}

413 Research in the Human Evolutionary Genetics lab is funded by the Institut Pasteur,

414 the Centre National de la Recherche Scientifique (CNRS), and the Fondation pour la

415 Recherche Médicale (Equipe FRM DEQ20180339214).

\section{Biosketch.}

419 Dr Maxime Rotival obtained his PhD in statistical genetics in 2011 from University of 420 Paris-Sud/XI. His PhD work regarded the genetic determinants of monocyte gene 421 expression in humans and their contribution to metabolic and cardiovascular 422 disorders. He did his first postdoc at Imperial College London, where he studied the

423 genetics of macrophage dysfunction in a rat model of chronic kidney inflammatory 424 disease, before joining the Human Evolutionary Genetics laboratory of Institut 425 Pasteur (CNRS UMR2000) in 2014. There he studied how genetic variants that alter 426 response to immune stimuli have contributed to the adaptation of modern human 427 population to pathogens present in their environment. He now has a researcher 428 position at institut Pasteur and is interested in the contribution of genetic diversity to 429 the inter-individual variability in innate immune response and how selective 430 pressures induced by pathogens have shaped this diversity. His work was awarded 431 with the Julia Bodmer Award for Young Scientists from the European Federation for 432 Immunogenetics in 2018.

\section{References}

435 1. Brodin P, Davis MM. Human immune system variation. Nat Rev Immunol. 436 2017;17(1):21-29.

437 2. Piasecka B, Duffy D, Urrutia A, et al. Distinctive roles of age, sex, and 438 genetics in shaping transcriptional variation of human immune responses to 439 microbial challenges. Proc Natl Acad Sci U S A. 2018;115(3):E488-E497. 
3. Alpert A, Pickman Y, Leipold M, et al. A clinically meaningful metric of immune age derived from high-dimensional longitudinal monitoring. Nat Med. 2019;25(3):487-495.

4. Brodin P, Jojic V, Gao T, et al. Variation in the human immune system is largely driven by non-heritable influences. Cell. 2015;160(1-2):37-47.

5. Netea MG, Joosten LA, Latz E, et al. Trained immunity: A program of innate immune memory in health and disease. Science. 2016;352(6284):aaf1098.

6. Barreiro LB, Tailleux L, Pai AA, Gicquel B, Marioni JC, Gilad Y. Deciphering the genetic architecture of variation in the immune response to Mycobacterium tuberculosis infection. Proc Natl Acad Sci U S A. 2012;109(4):1204-1209.

7. Lee $\mathrm{MN}, \mathrm{Ye} \mathrm{C}$, Villani $\mathrm{AC}$, et al. Common genetic variants modulate pathogen-sensing responses in human dendritic cells. Science. 2014;343(6175):1246980.

8. Fairfax BP, Humburg P, Makino $S$, et al. Innate immune activity conditions the effect of regulatory variants upon monocyte gene expression. Science. 2014;343(6175):1246949.

9. Quach H, Rotival M, Pothlichet J, et al. Genetic Adaptation and Neandertal Admixture Shaped the Immune System of Human Populations. Cell. 2016;167(3):643-656 e617.

10. Patin E, Quintana-Murci L. The demographic and adaptive history of central African hunter-gatherers and farmers. Curr Opin Genet Dev. 2018;53:90-97.

11. Nédelec Y, Sanz J, Baharian G, et al. Genetic Ancestry and Natural Selection Drive Population Differences in Immune Responses to Pathogens. Cell. 2016;167(3):657-669 e621.

12. Kim-Hellmuth S, Bechheim M, Putz B, et al. Genetic regulatory effects modified by immune activation contribute to autoimmune disease associations. Nat Commun. 2017;8(1):266.

13. Visscher PM, Wray NR, Zhang Q, et al. 10 Years of GWAS Discovery: Biology, Function, and Translation. Am J Hum Genet. 2017;101(1):5-22.

14. Pal LR, Yu CH, Mount SM, Moult J. Insights from GWAS: emerging landscape of mechanisms underlying complex trait disease. BMC Genomics. 2015;16 Suppl 8:S4.

15. Visel A, Rubin EM, Pennacchio LA. Genomic views of distant-acting enhancers. Nature. 2009;461(7261):199-205.

16. Gallagher MD, Chen-Plotkin AS. The Post-GWAS Era: From Association to Function. Am J Hum Genet. 2018;102(5):717-730.

17. Manolio TA, Collins FS, Cox NJ, et al. Finding the missing heritability of complex diseases. Nature. 2009;461(7265):747-753. 
18. Auer PL, Lettre G. Rare variant association studies: considerations, challenges and opportunities. Genome Med. 2015;7(1):16.

19. Wainschtein $P$, Jain $D P$, Yengo $L$, et al. Recovery of trait heritability from whole genome sequence data. bioRxiv. 2019:588020.

20. Hunt KA, Mistry V, Bockett NA, et al. Negligible impact of rare autoimmunelocus coding-region variants on missing heritability. Nature. 2013;498(7453):232-235.

21. Kellis M, Wold B, Snyder MP, et al. Defining functional DNA elements in the human genome. Proc Natl Acad Sci U S A. 2014;111(17):6131-6138.

22. Roadmap Epigenomics Consortium, Kundaje A, Meuleman W, et al. Integrative analysis of 111 reference human epigenomes. Nature. 2015;518(7539):317-330.

23. FANTOM Consortium, the RP, Clst, et al. A promoter-level mammalian expression atlas. Nature. 2014;507(7493):462-470.

24. Gondor A, Ohlsson R. Enhancer functions in three dimensions: beyond the flat world perspective. F1000Res. 2018;7.

25. Andersson R, Gebhard C, Miguel-Escalada I, et al. An atlas of active enhancers across human cell types and tissues. Nature. 2014;507(7493):455461.

26. ENCODE consortium, An integrated encyclopedia of DNA elements in the human genome. Nature. 2012;489(7414):57-74.

27. Core LJ, Martins AL, Danko CG, Waters CT, Siepel A, Lis JT. Analysis of nascent RNA identifies a unified architecture of initiation regions at mammalian promoters and enhancers. Nat Genet. 2014;46(12):1311.

28. Stunnenberg HG, International Human Epigenome C, Hirst M. The International Human Epigenome Consortium: A Blueprint for Scientific Collaboration and Discovery. Cell. 2016;167(5):1145-1149.

29. Chen L, Ge B, Casale FP, et al. Genetic Drivers of Epigenetic and Transcriptional Variation in Human Immune Cells. Cell. 2016;167(5):13981414 e1324.

30. Schmitt AD, Hu M, Jung I, et al. A Compendium of Chromatin Contact Maps Reveals Spatially Active Regions in the Human Genome. Cell Rep. 2016;17(8):2042-2059.

31. Javierre BM, Burren OS, Wilder SP, et al. Lineage-Specific Genome Architecture Links Enhancers and Non-coding Disease Variants to Target Gene Promoters. Cell. 2016;167(5):1369-1384 e1319.

32. Lecellier $\mathrm{CH}$, Wasserman WW, Mathelier A. Human Enhancers Harboring Specific Sequence Composition, Activity, and Genome Organization Are Linked to the Immune Response. Genetics. 2018;209(4):1055-1071. 
33. Arner E, Daub CO, Vitting-Seerup K, et al. Transcribed enhancers lead waves of coordinated transcription in transitioning mammalian cells. Science.

520 2015;347(6225):1010-1014.

34. Belton JM, McCord RP, Gibcus JH, Naumova N, Zhan Y, Dekker J. Hi-C: a

522

523

524

525

526

527

528

529

530

531

532

533

534

535

536

537

538

539

540

541

542

543

544

545

546

547

548

549

550

551

552

553 comprehensive technique to capture the conformation of genomes. Methods. 2012;58(3):268-276.

35. Dekker J, Marti-Renom MA, Mirny LA. Exploring the three-dimensional organization of genomes: interpreting chromatin interaction data. Nat Rev Genet. 2013;14(6):390-403.

36. Delaneau O, Zazhytska M, Borel C, et al. Intra- and inter-chromosomal chromatin interactions mediate genetic effects on regulatory networks. bioRxiv. 2017:171694.

37. Fanucchi S, Fok ET, Dalla E, et al. Immune genes are primed for robust transcription by proximal long noncoding RNAs located in nuclear compartments. Nat Genet. 2019;51(1):138-150.

38. Hnisz D, Abraham BJ, Lee TI, et al. Super-enhancers in the control of cell identity and disease. Cell. 2013;155(4):934-947.

39. Song W, Ovcharenko I. Dichotomy in redundant enhancers points to presence of initiators of gene regulation. BMC Genomics. 2018;19(1):947.

40. Lynch KW. Consequences of regulated pre-mRNA splicing in the immune system. Nat Rev Immunol. 2004;4(12):931-940.

41. Kornblihtt AR, Schor IE, Allo M, Dujardin G, Petrillo E, Munoz MJ. Alternative splicing: a pivotal step between eukaryotic transcription and translation. Nat Rev Mol Cell Biol. 2013;14(3):153-165.

42. Park E, Pan Z, Zhang Z, Lin L, Xing Y. The Expanding Landscape of Alternative Splicing Variation in Human Populations. Am J Hum Genet. 2018;102(1):11-26.

43. Ray $\mathrm{D}$, Kazan $\mathrm{H}$, Cook KB, et al. A compendium of RNA-binding motifs for decoding gene regulation. Nature. 2013;499(7457):172-177.

44. Lim KH, Ferraris L, Filloux ME, Raphael BJ, Fairbrother WG. Using positional distribution to identify splicing elements and predict pre-mRNA processing defects in human genes. Proc Natl Acad Sci U S A. 2011;108(27):1109311098.

45. Van Nostrand EL, Freese P, Pratt GA, et al. A Large-Scale Binding and Functional Map of Human RNA Binding Proteins. bioRxiv. 2018:179648.

46. Gebert LFR, MacRae IJ. Regulation of microRNA function in animals. Nat Rev Mol Cell Biol. 2019;20(1):21-37.

47. Bartel DP. MicroRNAs: target recognition and regulatory functions. Cell. 2009;136(2):215-233. 
48. Mehta A, Baltimore D. MicroRNAs as regulatory elements in immune system logic. Nat Rev Immunol. 2016;16(5):279-294.

49. Pai AA, Baharian G, Page Sabourin A, et al. Widespread Shortening of 3'

564 Untranslated Regions and Increased Exon Inclusion Are Evolutionarily Conserved Features of Innate Immune Responses to Infection. PLoS Genet. 2016;12(9):e1006338.

50. Neilsen CT, Goodall GJ, Bracken CP. IsomiRs--the overlooked repertoire in the dynamic microRNAome. Trends Genet. 2012;28(11):544-549.

51. Siddle KJ, Tailleux L, Deschamps $M$, et al. bacterial infection drives the expression dynamics of microRNAs and their isomiRs. PLoS Genet. 2015;11(3):e1005064.

52. Nejad C, Pillman KA, Siddle KJ, et al. miR-222 isoforms are differentially regulated by type-I interferon. RNA. 2018;24(3):332-341.

53. Yu F, Pillman KA, Neilsen CT, et al. Naturally existing isoforms of miR-222 have distinct functions. Nucleic Acids Res. 2017;45(19):11371-11385.

54. Stranger BE, Montgomery SB, Dimas AS, et al. Patterns of cis regulatory variation in diverse human populations. PLoS Genet. 2012;8(4):e1002639.

55. Montgomery SB, Sammeth M, Gutierrez-Arcelus M, et al. Transcriptome genetics using second generation sequencing in a Caucasian population. Nature. 2010;464(7289):773-777.

56. Pickrell JK, Marioni JC, Pai AA, et al. Understanding mechanisms underlying human gene expression variation with RNA sequencing. Nature. 2010;464(7289):768-772.

57. Lappalainen T, Sammeth M, Friedlander MR, et al. Transcriptome and genome sequencing uncovers functional variation in humans. Nature. 2013;501(7468):506-511.

58. Battle A, Mostafavi S, Zhu X, et al. Characterizing the genetic basis of transcriptome diversity through RNA-sequencing of 922 individuals. Genome Res. 2014;24(1):14-24.

59. Spielman RS, Bastone LA, Burdick JT, Morley M, Ewens WJ, Cheung VG. Common genetic variants account for differences in gene expression among ethnic groups. Nat Genet. 2007;39(2):226-231.

60. Saha A, Battle A. False positives in trans-eQTL and co-expression analyses arising from RNA-sequencing alignment errors. F1000Res. 2018;7:1860.

61. Võsa U, Claringbould A, Westra H-J, et al. Unraveling the polygenic architecture of complex traits using blood eQTL meta-analysis. bioRxiv. 2018:447367.

62. Montgomery SB, Dermitzakis ET. From expression QTLs to personalized transcriptomics. Nat Rev Genet. 2011;12(4):277-282. 
63. Consortium GT, Laboratory DA, Coordinating Center -Analysis Working G, et al. Genetic effects on gene expression across human tissues. Nature. 2017;550(7675):204-213.

64. Schmiedel BJ, Singh D, Madrigal A, et al. Impact of Genetic Polymorphisms on Human Immune Cell Gene Expression. Cell. 2018;175(6):1701-1715 e1716.

65. Nedelec Y, Sanz J, Baharian G, et al. Genetic Ancestry and Natural Selection Drive Population Differences in Immune Responses to Pathogens. Cell. 2016;167(3):657-669 e621.

66. Alasoo K, Rodrigues J, Mukhopadhyay S, et al. Shared genetic effects on chromatin and gene expression indicate a role for enhancer priming in immune response. Nat Genet. 2018;50(3):424-431.

67. Johnson CM, Lyle EA, Omueti KO, et al. Cutting edge: A common polymorphism impairs cell surface trafficking and functional responses of TLR1 but protects against leprosy. J Immunol. 2007;178(12):7520-7524.

68. van der Wijst MGP, Brugge $H$, de Vries $D H$, et al. Single-cell RNA sequencing identifies celltype-specific cis-eQTLs and co-expression QTLs. Nat Genet. 2018;50(4):493-497.

69. Li YI, van de Geijn B, Raj A, et al. RNA splicing is a primary link between genetic variation and disease. Science. 2016;352(6285):600-604.

70. Garieri M, Delaneau O, Santoni F, et al. The effect of genetic variation on promoter usage and enhancer activity. Nat Commun. 2017;8(1):1358.

71. Ye CJ, Chen J, Villani AC, et al. Genetic analysis of isoform usage in the human anti-viral response reveals influenza-specific regulation of ERAP2 transcripts under balancing selection. Genome Res. 2018;28(12):1812-1825.

72. Alasoo K, Rodrigues J, Danesh J, Freitag DF, Paul DS, Gaffney DJ. Genetic effects on promoter usage are highly context-specific and contribute to complex traits. Elife. 2019;8.

73. Sams AJ, Dumaine A, Nedelec Y, et al. Adaptively introgressed Neandertal haplotype at the OAS locus functionally impacts innate immune responses in humans. Genome Biol. 2016;17(1):246.

74. Odhams CA, Cunninghame Graham DS, Vyse TJ. Profiling RNA-Seq at multiple resolutions markedly increases the number of causal eQTLs in autoimmune disease. PLoS Genet. 2017;13(10):e1007071.

75. Galarza-Munoz G, Briggs FBS, Evsyukova I, et al. Human Epistatic Interaction Controls IL7R Splicing and Increases Multiple Sclerosis Risk. Cell. 2017;169(1):72-84 e13. 
633

634

635

636

637

638

639

640

641

642

643

644

645

646

647

648

649

650

651

652

653

654

655

656

657

658

659

660

661

662

663

664

665

666

667

668

669

670

76. Lappalainen T, Sammeth M, Friedlander MR, et al. Transcriptome and genome sequencing uncovers functional variation in humans. Nature. 2013;501(7468):506-511.

77. Huan T, Rong J, Liu C, et al. Genome-wide identification of microRNA expression quantitative trait loci. Nat Commun. 2015;6:6601.

78. Siddle KJ, Deschamps M, Tailleux L, et al. A genomic portrait of the genetic architecture and regulatory impact of microRNA expression in response to infection. Genome Res. 2014;24(5):850-859.

79. Budach S, Heinig M, Marsico A. Principles of microRNA Regulation Revealed Through Modeling microRNA Expression Quantitative Trait Loci. Genetics. 2016;203(4):1629-1640.

80. Wilk G, Braun R. regQTLs: Single nucleotide polymorphisms that modulate microRNA regulation of gene expression in tumors. PLoS Genet. 2018;14(12):e1007837.

81. Lord J, Gallone G, Short PJ, et al. Pathogenicity and selective constraint on variation near splice sites. Genome Res. 2018.

82. Farh KK, Marson A, Zhu J, et al. Genetic and epigenetic fine mapping of causal autoimmune disease variants. Nature. 2015;518(7539):337-343.

83. Chun S, Casparino A, Patsopoulos NA, et al. Limited statistical evidence for shared genetic effects of eQTLs and autoimmune-disease-associated loci in three major immune-cell types. Nat Genet. 2017;49(4):600-605.

84. Wang X, Goldstein DB. Enhancer redundancy predicts gene pathogenicity and informs complex disease gene discovery. bioRxiv. 2018:459123.

85. Hormozdiari F, van de Bunt M, Segre AV, et al. Colocalization of GWAS and eQTL Signals Detects Target Genes. Am J Hum Genet. 2016;99(6):12451260.

86. Smith RP, Taher L, Patwardhan RP, et al. Massively parallel decoding of mammalian regulatory sequences supports a flexible organizational model. Nat Genet. 2013;45(9):1021-1028.

87. Arnold CD, Gerlach D, Stelzer C, Boryn LM, Rath M, Stark A. Genome-wide quantitative enhancer activity maps identified by STARR-seq. Science. 2013;339(6123):1074-1077.

88. Melnikov A, Murugan A, Zhang X, et al. Systematic dissection and optimization of inducible enhancers in human cells using a massively parallel reporter assay. Nature Biotechnology. 2012;30:271.

89. Tewhey R, Kotliar D, Park DS, et al. Direct Identification of Hundreds of Expression-Modulating Variants using a Multiplexed Reporter Assay. Cell. 2016;165(6):1519-1529. 
671 90. Cheung R, Insigne KD, Yao D, et al. A Multiplexed Assay for Exon

672

673

674

675

676

677

678

679

680

681

682

683

684

685

686

687

688

689

690

691

692

693

694

695

696

697

698

699

700

701

702 Recognition Reveals that an Unappreciated Fraction of Rare Genetic Variants Cause Large-Effect Splicing Disruptions. Mol Cell. 2019;73(1):183-194 e188.

91. Soemedi R, Cygan $\mathrm{KJ}$, Rhine $\mathrm{CL}$, et al. Pathogenic variants that alter protein code often disrupt splicing. Nat Genet. 2017;49(6):848-855.

92. Rabani M, Pieper L, Chew GL, Schier AF. A Massively Parallel Reporter Assay of 3' UTR Sequences Identifies In Vivo Rules for mRNA Degradation. Mol Cell. 2017;68(6):1083-1094 e1085.

93. Vainberg Slutskin I, Weingarten-Gabbay S, Nir R, Weinberger A, Segal E. Unraveling the determinants of microRNA mediated regulation using a massively parallel reporter assay. Nat Commun. 2018;9(1):529.

94. Movva R, Greenside P, Shrikumar A, Kundaje A. Deciphering regulatory DNA sequences and noncoding genetic variants using neural network models of massively parallel reporter assays. bioRxiv. 2018:393926.

95. Zhou J, Theesfeld CL, Yao K, Chen KM, Wong AK, Troyanskaya OG. Deep learning sequence-based ab initio prediction of variant effects on expression and disease risk. Nat Genet. 2018;50(8):1171-1179.

96. Xiong HY, Alipanahi B, Lee LJ, et al. RNA splicing. The human splicing code reveals new insights into the genetic determinants of disease. Science. 2015;347(6218):1254806.

97. Finucane HK, Bulik-Sullivan B, Gusev A, et al. Partitioning heritability by functional annotation using genome-wide association summary statistics. Nat Genet. 2015;47(11):1228-1235.

98. Finucane HK, Reshef YA, Anttila V, et al. Heritability enrichment of specifically expressed genes identifies disease-relevant tissues and cell types. Nat Genet. 2018;50(4):621-629.

99. Porcu E, Rüeger S, Lepik K, Santoni FA, Reymond A, Kutalik Z. Mendelian Randomization integrating GWAS and eQTL data reveals genetic determinants of complex and clinical traits. bioRxiv. 2019:377267.

100. Nelson MR, Tipney H, Painter JL, et al. The support of human genetic evidence for approved drug indications. Nat Genet. 2015;47(8):856-860. 